\title{
MECANISMOS LEGAIS DE CONTROLE DA SEGURANÇA do MEDICAMENTO
}

LEGAL MECHANISMS OF MEDICINE'S SAFETY CONTROL

Felix J. Rosenberg(*)

\section{RESUMO}

O controle da segurança dos medicamentos é realizado através de leis nacionais interagindo no cenário mundial, pois cada país apresenta características específicas.

Para a análise desse controle, no Brasil, há vários aspectos essenciais: a viabilidade legal, ou seja, o conjunto de leis vigentes no país que regulam esta matéria; a viabilidade econômica e financeira, particularmente, no Brasil, com a descentralização do sistema de saúde; a viabilidade política, a qual contrasta muitos interesses. A respeito da viabilidade política, discorrese sobre dois temas: os programas implementados e a criação da ANVISA ligada à fiscalização e ao controle de qualidade e a legislação sanitária e os problemas relacionados para aplicação em diversos países.

O último aspecto essencial refere-se às estratégias de atuação da OMS e OPAS, organismos internacionais, em relação à legislação nacional, visando-se à sua harmoniosa convivência.

\section{Palavras-chave}

Medicamentos, Organismos Internacionais, Vigilância Sanitária.

\section{ABSTRACT}

The control of the safety of medicines is regulated by national laws interacting in the world scenery, because each country has specific characteristics.

(*) Ex-diretor do Instituto Nacional de Controle de Qualidade em Saúde. Fundação Oswaldo Cruz, Ministério da Saúde, Brasil. 
In Brazil, several important aspects entail the analysis of this control: the legal viability, in other words, the bulk of national effective laws regulating this subject; the economical and financial viability, particularly regarding the decentralized health system; the political viability, referring to the many counter interests. Regarding the political viability, two aspects are detailed. First, the previously implemented programs and the creation of ANVS (National Agency of Sanitary Surveillance), which is in charge of the fiscalization and the quality control. Second, the Sanitary International Law and the difficulties related to its enforcement in several countries.

The last essential aspect refers to the compatibility and harmonization of the national legislation to the WHO (World Health Organization) and PAHO (Pan American Health Organization) strategies and acts.

\section{Key words}

Medicine, International Organizations, World Health Organization, PAHO, Sanitary Surveillance, Health Care.

\section{INTRODUÇÃO}

Os mecanismos internacionais para o controle da segurança dos medicamentos não são supranacionais. Eles estão baseados em disposições legais nacionais e na sua articulação harmônica entre as nações. Neste sentido, é fundamental lembrar que os interesses e necessidades dos paises, apesar do mundo globalizado, não são iguais.

Dividiremos o assunto em pauta em quatro aspectos, utilizando, a maneira de exemplo, a situação atual no Brasil: em conseqüência, eles têm de estar baseados, como primeira premissa, em disposiçōes legais nacionais e na sua articulação harmônica entre as nações.

1. A disponibilidade de mecanismos legais (Viabilidade Legal).

2. A disponibilidade de recursos materiais para sua implementação (Viabilidade Econômica).

3. A disponibilidade de recursos políticos para sua implementação (Viabilidade Política).

4. O papel dos organismos internacionais na harmonização e na aplicação da legislação.

\section{A DISPONIBILIDADE DE MECANISMOS LEGAIS (VIABILIDADE LEGAL)}

O arcabouço legal disponivel no Brasil para o controle da segurança dos medicamentos, como na maioria dos países que dispõem de um certo grau de desenvolvimento industrial e/ou social, é amplo e suficiente para garantir que produtos químicos e biológicos de uso terapêutico ou preventivo em seres 
humanos sejam seguros quanto ao efeito desejado e quanto a ausência (ou a presença reduzida, controlável e conhecida) de efeitos indesejáveis.

Basicamente, estes instrumentos visam:

a) à disponibilidade pelo Estado de uma autoridade competente na área da segurança dos medicamentos. No Brasil, esta autoridade é denominada Sistema Nacional de Vigilância Sanitária (SNVS), sendo seus componentes e estrutura descritos na Portaria n. 1.565/94, amparada na Lei n. 8.080/90, também conhecida como Lei Orgânica da Saúde. A coordenação do Sistema, em âmbito federal, encontra-se situada na Agência Nacional de Vigilância Sanitária (ANVS) do Ministério da Saúde, criada pelo Decreto n. 3.029, de 16 de abril de 1999;

b) à avaliação e aprovação de todos os aspectos relacionados à eficácia e segurança do produto antes de sua comercialização perante autoridade nacional competente. Os mecanismos de registro de medicamentos estão previstos, em forma detalhada, na Lei n. 6.360 , de 23 de setembro de 1976, e em seu instrumento regulador, o Decreto $n$. 79.094/ 77. Cabe acrescentar a recente Lei n. 9.787/99, que regula o registro e comercialização dos medicamentos genéricos, em substituição ao Decreto n. 793/93;

c) ao cumprimento da indústria de medicamentos com os requisitos das Boas Práticas de Fabricação (BPF). Este cumprimento, no Brasil, está sujeito a inspeção por fiscais do SNVS, de acordo com procedimento aprovado em plano regional pelo MERCOSUL, baseado no guia GMP/92, publicado pela OMS;

d) à adequação das condições de armazenamento, distribuição e comercialização dos medicamentos aos requisitos técnicos necessários para preservar a qualidade dos medicamentos. Os procedimentos de inspeção na cadeia de distribuição de medicamentos estão previstos na Lei n. 5.991/73, regulamentada pelo Decreto n. 74.170/74;

e) à comercialização dos lotes de medicamentos com qualidade estabelecida e aprovada em ocasião do registro. Os procedimentos de controle da qualidade de medicamentos antes e após o registro, inclusive dos produtos no mercado, estão previstos na Lei n. 6.437/77;

f) à disponibilização de toda informação com relação aos riscos e benefícios de qualquer medicamento ao público. A legislação sobre o conteúdo da rotulagem de medicamentos, a propaganda e o direito do consumidor encontram-se na Lei n. 6.360/76, no Decreto n. 79.094/77 e no Código de Defesa do Consumidor, implementado através da Lei n. $8.072 / 90$.

\section{A DISPONIBILIDADE DE RECURSOS MATERIAIS PARA A IMPLEMENTAÇÃO DA LEGISLAÇÃO EM VIGOR (VIABILIDADE ECONỐMICA E FINANCEIRA)}

Apesar da cada vez mais frágil a situação dos recursos financeiros disponiveis para o Estado brasileiro, pode-se dizer que, estrategicamente 
distribuidos e corretamente aplicados, os recursos atualmente disponíveis pelo setor de saúde permitiriam uma satisfatória implementação da legislação sanitária em vigor. Contribuiria para tal fim, em forma determinante, a estratégia de descentralização de acordo com o previsto pela Lei n. 8.080/ 90, criadora do Sistema Único de Saúde.

Resumidamente, a estrutura descentralizada pertinente ao assunto em pauta consiste em:

1. O Sistema Nacional de Vigilância Sanitária, integrado pela Agência Nacional de Vigilância Sanitária, do Ministério da Saúde, em âmbito federal e pelas coordenações de vigilância sanitária das secretarias estaduais e municipais de saúde. Em forma esquemática, e de acordo com a distribuição de tarefas designadas no arcabouço legal e infralegal vigente, caberia, em princípio, à ANVS (isto é, em relação à União) as responsabilidades pela coordenação do sistema, pela concessão dos registros dos produtos e pela fiscalização dos portos, aeroportos e fronteiras; as vigilâncias sanitárias das secretarias estaduais de saúde têm como uma de suas atribuições principais as inspeções das indústrias farmacêuticas para verificar o cumprimento das BPFs; e às vigilâncias sanitárias municipais caberia, prioritariamente, as inspeções nos serviços de saúde e na comercialização de medicamentos. Está claro, não obstante, na Lei n. 8.080/90, que cada instância superior de governo atua em forma complementar à instância descentralizada. Desta forma, a maior ou menor atuação de cada Secretaria Municipal de Saúde no processo dependerá do seu maior ou menor grau de desenvolvimento na gestão do setor.

2. A Rede Brasileira de Laboratórios Analíticos em Saúde (REBLAS), integrada pelo Instituto Nacional de Controle de Qualidade em Saúde (INCQS), do Ministério da Saúde, os laboratórios centrais estaduais de Saúde Pública (LACEN) e outros laboratórios prestadores de serviços. Neste esquema, o INCQS atuaria como cabeça da rede de laboratórios de referência sendo responsável, junto com os LACENs, tecnicamente capacitados, pelo controle da qualidade dos medicamentos, nas modalidades prévia (em amostras submetidas como requisito no processo de registro), de controle (em amostras de lotes de produção, durante o primeiro ano após a colocação destes no mercado) e fiscal (em amostras apreendidas aleatoriamente ou por suspeita na cadeia de distribuição).

3. A Farmacovigilância: Apesar da existência de alguns núcleos de farmacovigilância, pesquisa e informação de medicamentos distribuidos em diversas Universidades e Centros de Pesquisa no país, o Sistema Nacional de Vigilância Sanitária não tem desenvolvido, até o presente, nenhum esforço para sistematizar a informação gerada. Tendo em consideração que o conhecimento básico do componente fundamental para a segurança dos medicamentos encontra-se em instituições de pesquisa que não dependem diretamente do Governo, é bem possivel que esta omissão do Sistema não seja tão somente casual. 


\section{A DISPONIBILIDADE DE RECURSOS POLÍTICOS (VIABILIDADE POLÍTICA) NA REALIDADE BRASILEIRA}

A questão dos medicamentos, como é bem sabido, envolve enormes interesses vinculados à exploração econômica e/ou política de sua deman$\mathrm{da}$, em muitos casos incrementada além das verdadeiras necessidades da população. Transformado em commodity, o papel real do medicamento como elemento de prevenção ou recuperação da saúde, no contexto do poder real, é secundário.

Entrar na discussão sobre a ética do medicamento não é objeto desta reunião nem sujeito do meu conhecimento. A vasta discussão sobre o uso racional de medicamentos é mais do que eloqüente neste sentido, na mesma proporção, por sinal, em que os meios de comunicação de massa a omitem.

Pretendo, sim, apresentar, através de alguns exemplos que são da minha experiência e conhecimento pessoal, qual tem sido o comportamento político das autoridades nacionais de saúde a respeito da implementação objetiva e neutra da legislação sobre segurança dos medicamentos.

Em primeiro lugar, é fundamental deixar absolutamente claro que a política global de distribuição gratuita de medicamentos tem sido se não ideal quanto às necessidades da população, pelo menos digna de reconhecimento, em particular quando comparada com outros países de características semelhantes ao nosso. Desta forma, a análise política que aqui será feita não pode, sob hipótese alguma, ser interpretada como uma crítica aos objetivos dos programas nacionais que, como o PNI, DST, AIDS e vários outros, têm batalhado durante anos para manter uma cobertura adequada de medicação e/ou vacinação.

\section{A ANVS e o Sistema Único de Saúde}

a) A Fiscalização

É por todos bem sabido que a segurança dos medicamentos no Brasil tem deixado a desejar. Um fator determinante para esta falência reside na alta rotatividade dos dirigentes: durante os últimos 6 anos, a Secretaria de Vigilância Sanitária/Ministério da Saúde teve sete secretários designados (mais alguns interinos). Uma média menor de um por ano!

A solução encontrada foi a criação da Agência Nacional de Vigilância Sanitária, à qual, aparentemente, seriam dados os instrumentos necessários para resolver problemas crônicos na área. Ela permite, de fato, um certo grau de flexibilização na ordenação e aplicação financeira, imprescindivel para gerenciar o órgão de inteligência sanitária do país, e determina, por decreto, a estabilidade dos seus máximos dirigentes. 
As altas autoridades nacionais foram procurar inspiração, para a criação da agência, no FDA. Copiaram, porém, o que o Sistema Americano tem de pior: sua estrutura federal independente, própria da história política e econômica dos EUA, reduzindo a um espaço secundário o que o Sistema Brasileiro tem de melhor: a Lei n. 8.080/90.

O Sistema Americano, respeitando a soberania de estados e municípios, dispõe, de fato, de três sistemas de vigilância sanitária, que operam em forma independente: o sistema municipal, para os produtos de circulação intramunicipal; o sistema estadual, para os produtos de circulação intraestadual; e o sistema federal, o FDA, para os produtos de circulação interestadual e internacional. Explica-se, assim, que em grandes cidades americanas operem estruturas paralelas de registro, fiscalização e controle da qualidade, cada uma delas atendendo ao setor estabelecido para sua atuação. Este sistema encontra-se atualmente em falência estrutural, obrigando - FDA a subcontratar serviços das instâncias estaduais, municipais e até de prestadores de serviços privados.

No Brasil, de acordo com o estabelecido pela Lei Orgânica de Saúde, a Vigilância Sanitária deve operar como um Sistema Único, no qual cada instância ou esfera de governo atenda às demandas do sistema nacional, em seu setor de atuação. Desta forma, cabem à esfera federal as ações de coordenação (incluindo, obviamente, a normalização, referência, informação etc.), atuando, complementarmente, em apoio às ações estaduais; estas atuam como coordenadores e referência para o setor estadual e, complementarmente, na operação no setor municipal, enquanto estas últimas seriam as responsáveis pela operação do Sistema, em função do seu grau de capacidade técnica e dos recursos disponíveis. A Norma Operacional Básica (NOB-96) estabelece o volume de recursos financeiros repassados para cada uma das responsabilidades alocadas, no contexto do Sistema.

De acordo com o texto do Decreto n. $3.029 / 99$ e analisando os primeiros passos dados pela ANVS, surgem sérias preocupações com relação à intenção do Ministério da Saúde em assumir de forma centralizada as ações mais relevantes do controle da segurança dos medicamentos, apesar do estabelecido na Lei Orgânica de Saúde.

Tanto do ponto de vista do aproveitamento dos recursos disponíveis, quanto da própria filosofia da descentralização como instrumento da democratização do poder público, esta decisão constituirá, se realmente implementada, um retrocesso em lugar de um avanço. Mas, certamente, as pressões nesse sentido não devem ser procuradas no campo meramente administrativo. Em todo caso está claro que, propositadamente ou não, trata-se de um ato de centralização do poder em instâncias decisivas do governo federal.

A alternativa viável para este processo, por parte do governo federal, é a de investir na elaboração, modernização, divulgação e implementação de 
um Código Sanitário Nacional; na elaboração e implementação de procedimentos para a avaliação do Sistema; nas ações de informação à população e, particularmente, no fortalecimento das estruturas executivas descentralizadas, estaduais e municipais.

b) O Controle da Qualidade

É por todos bem sabido que o controle da qualidade dos medicamentos no Brasil tem deixado a desejar. É importante destacar como fator determinante dessa situação que, entre 1985 e 1993, o INCQS, Instituto Nacional de Controle de Qualidade em Saúde, órgão da Fundação Oswaldo Cruz/ Ministério da Saúde, criado em 1981, teve seis Diretores, uma média de 1,4 ano/Diretor. O INCQS foi, inclusive, o único dos onze Institutos da FIOCRUZ a ter um Diretor eleito exonerado por ato oficial, sem justificativa aparente. Por sinal, não apenas um senão os dois únicos diretores eleitos: o primeiro, durante o governo Collor, o segundo, após cinco anos de exercício, em 1999. A partir de então o problema ficou muito mais fácil. Simplesmente foi abolida a eleição do diretor do INCQS, sendo seu diretor indicado pelo Presidente da Agência Nacional de Vigilância Sanitária, diferenciando-o assim da própria Presidencia e dos demais Institutos da FIOCRUZ, cujos dirigentes continuam a ser eleitos. Mais um ato de centralização do poder nessa área.

O Controle da qualidade dos medicamentos que estão no mercado é um processo complexo e lento. O laboratório deve estar preparado para analisar qualquer um dos princípios ativos existentes, freqüentemente, sua composição quali-quantitativa e, ocasionalmente, possíveis impurezas e contaminantes. Não é como controle de qualidade (tanto na indústria - controle de processo - quanto no controle oficial sistemático de lote) que, sendo a atividade rotineira e programada, a produtividade analítica pode ser muito maior. Dessa forma, durante o período 94-98 o INCQS apesar de quadruplicar o número de lotes de medicamentos analisados anualmente, de cerca de 90 para quase 400 , ainda ficou muito aquém da necessidade nacional, estimada em não menos de três mil lotes/ano. No mesmo período, o número de lotes de vacinas controlados anualmente, esses em forma rotineira, oscilou em torno de dois mil lotes, representando a totalidade das vacinas distribuídas pelo Estado para o Programa Nacional de Imunizações.

Assim sendo, o INCQS acreditou na necessidade do desenvolvimento dos recursos humanos e materiais nos Laboratórios das Secretarias Estaduais de Saúde, com o duplo propósito de incrementar a capacidade laboratorial instalada e de aproximar o usuário à disponibilidade do serviço. Desde o início desse período, foi dada grande ênfase na necessidade de implementar sistemas de gerenciamento da qualidade nos laboratórios públicos com a finalidade de incrementar a confiabilidade nos resultados analíticos.

Como conseqüência dessa necessidade, a ANVS acabou de publicar a Resolução $n$. 299, que estabelece a necessidade do credenciamento pelo 
INMETRO de todos os laboratórios prestadores de serviços para o setor saúde, tanto em âmbito público quanto privado. A medida é exemplar. Porém, se os laboratórios oficiais não receberem um apoio substancial para a implementação dos seus sistemas de gerenciamento da qualidade, muito pou$\cos$, além do INCQS, terão condições de realizar controles oficiais da qualidade dos medicamentos. A conseqüência mais provável dessa decisão é que o controle da qualidade de medicamentos fique reduzido e sob controle estrito de órgão federal e/ou que laboratórios privados credenciados sejam autorizados a participar de maneira mais intensa nas atividades fiscais, uma das poucas atribuições que o Estado brasileiro está ainda guardando para si.

\section{A Legislação Sanitária e os Amigos}

$\mathrm{Na}$ questāo do respeito à Lei, o Brasil navega entre duas vertentes muito populares em vários países dessa região do mundo: a versão nepótica e demagógica, mais light, de "Para os amigos tudo; para os inimigos a Lei" e a versão mais fascista: "Para os amigos tudo, para os inimigos nem a Lei".

Da segunda versão, existem vários exemplos especificamente referidos àqueles processos de registro de medicamentos que, por uma razão ou outra, não interessam aos agentes do poder real que estabelecem as regras no Sistema. Estes processos descansam, nos meandros da burocracia, os sonhos dos justos. Mas, não é esse o objeto desta apresentação.

Muito pior são os vários exemplos de registros sumários de medicamentos pressionados por lobbies comerciais ou políticos poderosos, inclusive por meio de canais e procedimentos não previstos nos regulamentos vigentes e apesar de pareceres técnicos oficiais em contrário. Alguns destes pareceres contrários foram considerados até uma afronta aos "interesses nacionais" ou "aquém das necessidades de um mundo globalizado".

Um outro exemplo do uso arbitrário da lei está dado pela decisão de năo atender aos requisitos da legislação de controle da segurança para as compras de produtos por parte do Estado.

Uns 5 a 6 anos atrás, um assessor legal do Ministério da Saúde interpretou através de um parecer relativo à compra de hemoderivados que a Lei n. 6.360/76 não se aplicaria às compras realizadas pelo Estado já que "o Estado não iria se conceder registro de importação e comercialização a si próprio". Esta aberração juridica foi sustentada alguns anos depois pelo Programa Nacional de Imunizações (PNI), da Fundação Nacional de Saúde, para não ter de submeter as compras de vacinas (cujo custo varia anualmente em torno dos 100 milhões de dólares) ao requisito do registro no país. Um dos argumentos era que a exigência do cumprimento desse requisito legal poderia induzir à impugnação das licitações públicas (transcorridos cerca 
de três anos dessa discussão, ainda hoje não é fácil compreender este argumento). O outro argumento era, no mínimo, cínico: "o cumprimento dos requisitos legais no Brasil traduzir-se-ia em preços mais altos dos produtos". O menosprezo do papel da legislação sanitária e a mercantilização do medicamento pelo próprio Governo parecem-nos deploráveis.

Tendo em vista a posiçāo tecnicamente intransigente do INCQS, ao emitir laudos correspondentes aos lotes importados como "Insatisfatórios" por não constar o número de registro no país e por conterem rótulos e bulas em língua estrangeira e considerando a incapacidade do Ministério para resolver as constantes impugnações às quais suas licitações eram submetidas, o PNI/FNS tomou a decisão de delegar na OPAS todas as compras de vacinas produzidas no estrangeiro. Esse fato permite-nos analisar sinteticamente o papel dos Organismos Internacionais no controle da segurança dos medicamentos, pelo menos, no Brasil.

\section{O PAPEL DOS ORGANISMOS INTERNACIONAIS NA HARMONIZAÇÃO E NA APLICAÇÃO DA LEGISLAÇÃO}

Na área que nos ocupa, tanto a Organização Mundial da Saúde (OMS) quanto seu organismo regional para as Américas, a Organização Pan-Americana da Saúde (OPAS), vêm desenvolvendo um importante papel de cooperação técnica através da assessoria para a produção, controle da segurança e o uso racional de medicamentos. Uma das atividades destacadas é sua participaçāo, em conjunto com outros organismos, no Programa Global de Vacinas.

Dentre suas estratégias de atuação destaca-se a tentativa de harmonizar a legislação sanitária entre os paises como um dos principais mecanismos para garantir o uso seguro de medicamentos, incluindo o combate dos medicamentos falsificados. Outro papel de transcendência tem sido o de distribuir, às vezes de forma gratuita, outras por meio de fundos rotatórios, medicamentos e, particularmente, vacinas para paises pobres e pequenos.

Recentemente, somando essa estratégia do fundo rotatório da OPAS às dificuldades do governo brasileiro para montar um processo ágil e adequado de licitações, ambas instituições decidiram juntar seus esforços numa iniciativa que, à primeira vista, pode parecer sumamente inteligente: a OPAS passava a adquirir todas as vacinas requeridas pelo Programa Nacional de Imunizaçōes do Brasil através do seu fundo rotatório. Mediante essa iniciativa, o governo do Brasil isentava-se de sua responsabilidade na aquisição das vacinas e a OPAS conseguia uma significativa redução dos preços devido ao elevado volume de compra do Brasil (como exemplo, o Brasil adquire mais de dois mil lotes de vacinas/ano enquanto os países normalmente beneficiários do fundo recebem entre dez e vinte lotes/ano), além de incorporar 
a seu orçamento local uma importante parcela advinda dos gastos de administração.

Sem querer entrar no mérito da atribuição dos organismos de cooperação técnica como administradores das compras de insumos de saúde em países do porte do Brasil, o conflito surgiu quando a OPAS e o governo brasileiro acordaram, apesar da oposição dos órgãos de controle técnico e regulatório na época, que estes produtos não requeriam registro no Brasil já que a OPAS "garantia" a origem e qualidade destes produtos. A OPAS, em conjunto com o setor correspondente da OMS e a anuência do $\mathrm{PNI} / \mathrm{MS}$, constituiu-se assim em um órgão de regulação sanitária supranacional, obrigando o país comprador a desobedecer aqueles regulamentos harmonizados cuja implementação é, por eles mesmos, "altamente recomendada".

A então secretária de Vigilância Sanitária foi exonerada pelo ministro, dentre outras razões, por sua oposição em emitir qualquer autorização a essas compras. Eu, ex-diretor do INCQS, fui exonerado alguns meses depois, dentre outras razōes, por ter assinado boletins analíticos declarando insatisfatórios lotes de produtos adquiridos pela OPAS, cujo $\mathrm{n}$. de registro não constava no rótulo e/ou cujo rótulo e bula não estavam redigidos em língua portuguesa e/ou cujo nome fantasia não obedecia à Lei n. 6.360/76 $e$, inclusive em alguns casos, cujos padrões de eficácia ou segurança estavam fora dos limites estabelecidos por normas nacionais e recomendações da própria OMS.

Para resolver esse "impasse", além da exoneração do diretor do INCQS, o Governo aproveitou o Decreto n. 3.029, de abril de 1999, para criar a Agência Nacional de Vigilância Sanitária e incluir um parágrafo que "legaliza" essa ilegalidade: "A Agência poderá dispensar de registro os imunobiológicos, inseticidas, medicamentos e outros insumos estratégicos, quando adquiridos por intermédio de organismos multilaterais internacionais, para uso em programas de saúde pública pelo Ministério da Saúde e suas entidades vinculadas".

Tal atitude tem severas implicações sanitárias, políticas e éticas. Do ponto de vista sanitário, utilizam-se produtos cuja eficácia e segurança não foram analisadas no contexto das condições geográficas, epidemiológicas e sociais de nosso país. Do ponto de vista político, abre-se mão da soberania nas decisões dos órgãos públicos técnicos e regulatórios nacionais. Quanto ao aspecto ético, um mesmo organismo, seja nacional ou internacional, não pode autorizar, comprar, distribuir, aplicar, controlar e avaliar qualquer produto que seja, menos ainda um medicamento. É justamente por isso que tanto as estruturas de Governo quanto as das organizações privadas segregam adequadamente as funções que são incompativeis ou conflitantes entre si.

O Decreto n. 3.181/99, que acaba de ser publicado, estabelece critérios extremadamente rígidos para o registro de medicamentos genéricos no 
país. São poucos os países do mundo que adotam exigências de estudos de bioequivalência in vivo para praticamente todos os genéricos de uso sistêmico e de autorização prévia para a mudança do fabricante de matéria prima. De fato, tais requisitos foram descartados, após um profundo debate, como elementos a serem incorporados no guia de prevenção de medicamentos falsificados, recentemente publicado pela OMS.

Para a indústria nacional tornar-se-á extremadamente difícil, a curto prazo, registrar medicamentos genéricos de maneira generalizada. Para o Governo Federal, entretanto, bastará aplicar o Decreto n. 3.029/99 e, à semelhança do que é feito em relação às vacinas e outros produtos de saúde, adquirir medicamentos genéricos, através do fundo rotatório de organismos internacionais, de países cujos requisitos de registro, técnica e politicamente mais razoáveis, não incorporem estas exigências.

Em todo caso, não deixaria de ser mais uma maneira peculiar de fazer e de respeitar a Lei. 